\title{
Traveling Wave Solutions of Nonlinear Evolution Equations Via the New Generalized $\left(G^{\prime} / G\right)$-Expansion Method
}

\author{
Md. Nur Alam, ${ }^{1, *}$, M. Ali Akbar ${ }^{2}$ \\ ${ }^{1}$ Department of Mathematics, Pabna University of Science and Technology, Bangladesh \\ ${ }^{2}$ Department of Applied Mathematics, University of Rajshahi, Bangladesh \\ *Corresponding Author: nuralam.pstu23@gmail.com
}

Copyright $(2013$ Horizon Research Publishing All rights reserved.

\begin{abstract}
The exact solutions of nonlinear evolution equations (NLEEs) play a crucial role to make known the internal mechanism of compound physical phenomena. In this article, we implement the new generalized ( $G^{\prime} / G$ )-expansion method for seeking the exact solutions of NLEEs via the Benjamin-Ono equation and achieve exact solutions involving parameters. Abundant traveling wave solutions with arbitrary parameters are successfully obtained by this method and the wave solutions are expressed in terms of the hyperbolic, trigonometric, and rational functions. It is shown that the new approach of generalized $\left(G^{\prime} / G\right)$ -expansion method is a powerful and concise mathematical tool for solving nonlinear evolution equations.
\end{abstract}

Keywords New Approach Of Generalized $\left(G^{\prime} / G\right)$ -Expansion Method, The Benjamin-Ono Equations, Homogeneous Balance, Traveling Wave Solutions, Nonlinear Evolution Equation

\section{Introduction}

Nonlinear wave phenomena appears in various scientific and engineering fields such as fluid mechanics, plasma physics, optical fibers, biophysics, geochemistry, electricity, propagation of shallow water waves, high-energy physics, condensed matter physics, quantum mechanics, elastic media, biology, solid state physics, chemical kinematics, chemical physics and so on. This is also noticed to arise in engineering, chemical and biological applications. The application of nonlinear traveling waves has been brought prosperity in the field of applied science. In order to understand better the nonlinear phenomena as well as further application in the practical life, it is important to seek their more exact travelling wave solutions. Essentially all the fundamental equations in physical sciences are nonlinear and, in general, such NLEEs are often very complicated to solve explicitly. The exact solutions of NLEEs play an important role in the study of nonlinear physical phenomena. Therefore, the powerful and efficient methods to find exact solutions of nonlinear equations still have drawn a lot of interest by diverse group of scientists. In the past three decades, there has been significant progress in the development of finding effective methods for obtaining exact solutions of NLEEs. These methods are the homogeneous balance method [1], the tanh-function method [2], the extended tanh-function method [3, 4], the Exp-function method [5-7], the sine-cosine method [8], the modified Exp-function method [9], the generalized Riccati equation [10], the Jacobi elliptic function expansion method [11, 12], the Hirota's bilinear method [13], the Miura transformation [14], the $\left(G^{\prime} / G\right)$ -expansion method [15-19], the novel $\left(G^{\prime} / G\right)$-expansion method $[20,21]$, the modified simple equation method [22, 23], the improved $\left(G^{\prime} / G\right)$-expansion method [24], the inverse scattering transform [25] and so on.

Recently, Naher and Abdullah [26] established a highly effective extension of the $\left(G^{\prime} / G\right)$ expansion method, called the new generalized $\left(G^{\prime} / G\right)$ expansion method to obtain exact traveling wave solutions of NLEEs. The aim of this article is to look for new study relating to the new generalized $\left(G^{\prime} / G\right)$ expansion method for solving the Benjamin-Ono equation to make obvious the effectiveness and usefulness of the method.

The outline of this paper is organized as follows: In Section 2, we give the description of the new generalized $\left(G^{\prime} / G\right)$ expansion method. In Section 3, we apply this method to the Benjamin-Ono equation, results and discussions and graphical representation of solutions. Conclusions are given in the last section.

\section{Description of the New Generalized $\left(G^{\prime} / G\right)$-Expansion Method}

Let us consider a general nonlinear PDE in the form

$$
\Phi\left(v, v_{t}, v_{x}, v_{x x}, v_{t t}, v_{t x}, \ldots\right),
$$


where $v=v(x, t)$ is an unknown function, $\Phi$ is a polynomial in $v(x, t)$ and its derivatives in which highest order derivatives and nonlinear terms are involved and the subscripts stand for the partial derivatives.

Step 1: We combine the real variables $x$ and $t$ by a complex variable $\eta$

$$
v(x, t)=v(\eta), \quad \eta=x \pm V t,
$$

where $v$ is the speed of the traveling wave. The traveling wave transformation (2) converts Eq. (1) into an ordinary differential equation (ODE) for $v=v(\eta)$ :

$$
\psi\left(v, v^{\prime}, v^{\prime \prime}, v^{\prime \prime \prime}, \cdots\right) \text {, }
$$

where $\psi$ is a polynomial of $v$ and its derivatives and the superscripts indicate the ordinary derivatives with respect to $\eta$.

Step 2: According to possibility, Eq. (3) can be integrated term by term one or more times, yields constant(s) of integration. The integral constant may be zero, for simplicity.

Step 3. Suppose the traveling wave solution of Eq. (3) can be expressed as follows:

$$
v(\eta)=\sum_{i=0}^{N} \alpha_{i}(d+M)^{i}+\sum_{i=1}^{N} \beta_{i}(d+M)^{-i},
$$

where either $\alpha_{N}$ or $\beta_{N}$ may be zero, but could be zero simultaneously, $\alpha_{i} \quad(i=0,1,2, \cdots, N)$ and $\beta_{i} \quad(i=1,2, \cdots, N)$ and $d$ are arbitrary constants to be determined and $M(\eta)$ is

$$
M(\eta)=\left(G^{\prime} / G\right)
$$

where $G=G(\eta)$ satisfies the following auxiliary nonlinear ordinary differential equation:

$$
A G G^{\prime \prime}-B G G^{\prime}-E G^{2}-C\left(G^{\prime}\right)^{2}=0,
$$

where the prime stands for derivative with respect to $\eta ; A$, $B, C$ and $E$ are real parameters.

Step 4: To determine the positive integer $N$, taking the homogeneous balance between the highest order nonlinear terms and the derivatives of the highest order appearing in Eq. (3).

Step 5: Substitute Eq. (4) and Eq. (6) including Eq. (5) into Eq. (3) with the value of $N$ obtained in Step 4, we obtain polynomials in $(d+M)^{N}(N=0,1,2, \cdots)$ and $(d+M)^{-N}$ $(N=0,1,2, \cdots)$. Subsequently, we collect each coefficient of the resulted polynomials to zero, yields a set of algebraic equations for $\alpha_{i}(i=0,1,2, \cdots, N)$ and $\beta_{i} \quad(i=1,2, \cdots, N)$, $d$ and $V$.

Step 6: Suppose that the value of the constants $\alpha_{i}$ $(i=0,1,2, \cdots, N), \beta_{i} \quad(i=1,2, \cdots, N), d$ and $V$ can be found by solving the algebraic equations obtained in Step 5. Since the general solutions of Eq. (6) are known to us, inserting the values of $\alpha_{i}(i=0,1,2, \cdots, N), \beta_{i} \quad(i=1,2, \cdots, N), d$ and
$V$ into Eq. (4), we obtain more general type and new exact traveling wave solutions of the nonlinear partial differential equation (1).

Step 7: Using the general solution of Eq. (6), we have the following solutions of Eq. (5):

Family 1: When $B \neq 0, \quad \omega=A-C \quad$ and $\Omega=B^{2}+4 E(A-C)>0$,

$$
M(\eta)=\left(\frac{G^{\prime}}{G}\right)=\frac{B}{2 \omega}+\frac{\sqrt{\Omega}}{2 \omega} \frac{C_{1} \sinh \left(\frac{\sqrt{\Omega}}{2 A} \eta\right)+C_{2} \cosh \left(\frac{\sqrt{\Omega}}{2 A} \eta\right)}{C_{1} \cosh \left(\frac{\sqrt{\Omega}}{2 A} \eta\right)+C_{2} \sinh \left(\frac{\sqrt{\Omega}}{2 A} \eta\right)}
$$

Family 2: When $B \neq 0, \quad \omega=A-C \quad$ and $\Omega=B^{2}+4 E(A-C)<0$,

$$
M(\eta)=\left(\frac{G^{\prime}}{G}\right)=\frac{B}{2 \omega}+\frac{\sqrt{-\Omega}}{2 \omega} \frac{-C_{1} \sin \left(\frac{\sqrt{-\Omega}}{2 A} \eta\right)+C_{2} \cos \left(\frac{\sqrt{-\Omega}}{2 A} \eta\right)}{C_{1} \cos \left(\frac{\sqrt{-\Omega}}{2 A} \eta\right)+C_{2} \sin \left(\frac{\sqrt{-\Omega}}{2 A} \eta\right)}
$$

Family 3: When $B \neq 0, \quad \omega=A-C \quad$ and $\Omega=B^{2}+4 E(A-C)=0$,

$$
M(\eta)=\left(\frac{G^{\prime}}{G}\right)=\frac{B}{2 \omega}+\frac{C_{2}}{C_{1}+C_{2} \eta}
$$

Family 4: When $B=0, \omega=A-C$ and $\Delta=\omega E>0$,

$$
M(\eta)=\left(\frac{G^{\prime}}{G}\right)=\frac{\sqrt{\Delta}}{\omega} \frac{C_{1} \sinh \left(\frac{\sqrt{\Delta}}{A} \eta\right)+C_{2} \cosh \left(\frac{\sqrt{\Delta}}{A} \eta\right)}{C_{1} \cosh \left(\frac{\sqrt{\Delta}}{A} \eta\right)+C_{2} \sinh \left(\frac{\sqrt{\Delta}}{A} \eta\right)}
$$

Family 5: When $B=0, \omega=A-C$ and $\Delta=\omega E<0$,

$$
M(\eta)=\left(\frac{G^{\prime}}{G}\right)=\frac{\sqrt{-\Delta}}{\omega} \frac{-C_{1} \sin \left(\frac{\sqrt{-\Delta}}{A} \eta\right)+C_{2} \cos \left(\frac{\sqrt{-\Delta}}{A} \eta\right)}{C_{1} \cos \left(\frac{\sqrt{-\Delta}}{A} \eta\right)+C_{2} \sin \left(\frac{\sqrt{-\Delta}}{A} \eta\right)}
$$

\section{Application of the Method}

In this section, we will present the new generalized $\left(G^{\prime} / G\right)$ expansion method to construct many new and more general traveling wave solutions of the Benjamin-Ono equation. Let us consider the Benjamin-Ono equation,

$$
v_{t}+h v_{x x}+v v_{x}=0 .
$$

We utilize the traveling wave variable $w(\eta)=v(x, t)$, $\eta=x-V t$, Eq. (19) is carried to an ODE

$$
-V w^{\prime}+h w^{\prime \prime}+\frac{1}{2}\left(w^{2}\right)^{\prime}=0 .
$$


Eq. (20) is integrable, therefore, integrating with respect to $\eta$ once yields:

$$
P-V w+h w^{\prime}+\frac{1}{2} w^{2}=0
$$

where $P$ is an integration constant which is to be determined.

Taking the homogeneous balance between highest order nonlinear term $w^{2}$ and linear term of the highest order $w^{\prime}$ in Eq. (14), we obtain $N=1$. Therefore, the solution of Eq. (14) is of the form:

$$
w(\eta)=\alpha_{0}+\alpha_{1}(d+M)+\beta_{1}(d+M)^{-1},
$$

where $\alpha_{0}, \alpha_{1}, \beta_{1}$ and $d$ are constants to be determined.

Substituting Eq. (15) together with Eqs. (5) and (6) into Eq. (14), the left-hand side is converted into polynomials in $(d+M)^{N}(N=0,1,2, \ldots \ldots$.$) and (d+M)^{-N}(N=1,2, \cdots)$. We collect each coefficient of these resulted polynomials to zero yields a set of simultaneous algebraic equations (for simplicity, the equations are not presented) for $\alpha_{0}, \alpha_{1}, \beta_{1}, d, P$ and $V$. Solving these algebraic equations with the help of computer algebra, we obtain following:

Set 1: $P=\frac{1}{2 A^{4}}\left(4 h^{2} d^{2} \omega^{2}+4 h^{2} B d \omega-4 h^{2} E \omega-2 a_{0} A h B+4 a_{0} A h C d+a_{0}^{2} A^{2}-4 a_{0} A^{2} h d\right)$,

$$
\alpha_{0}=\alpha_{0}, \quad V=-\frac{1}{A}\left(h B+2 h d \omega-a_{0} A\right), \quad d=d, \quad \alpha_{1}=0, \beta_{1}=-\frac{2 h}{A}\left(d^{2} \omega+B d-E\right)
$$

where $\omega=A-C, a_{0}, d, A, B, C, E$ are free parameters.

Set 2: $P=\frac{1}{2 A^{4}}\left(4 h^{2} d^{2} \omega^{2}-4 h^{2} E \omega+a_{0}^{2} A^{2}+2 a_{0} A h B+4 a_{0} A^{2} h d-4 a_{0} A h C d+4 h^{2} B d \omega\right)$,

$$
\alpha_{0}=\alpha_{0}, V=\frac{1}{A}\left(a_{0} A+h B+2 h d \omega\right), d=d, \beta_{1}=0, \alpha_{1}=\frac{2 h \omega}{A} .
$$

where $\omega=A-C, a_{0}, d, A, B, C, E$ are free parameters.

Set 3: $P=\frac{1}{2 A^{2}}\left(a_{0}^{2} A^{2}-16 h^{2} E \omega-4 h^{2} B^{2}\right), V=a_{0}, d=-\frac{B}{2 \omega}$

$$
\alpha_{0}=\alpha_{0}, \alpha_{1}=\frac{2 h \omega}{A}, \beta_{1}=\frac{h}{A \omega}\left(4 E \omega+B^{2}\right),
$$

where $\omega=A-C, V, A, B, C, E$ are free parameters.

For set 1, substituting Eq. (16) into Eq. (15), along with Eq. (7) and simplifying, yields following traveling wave solutions, if $C_{1}=0$ but $C_{2} \neq 0 ; C_{2}=0$ but $C_{1} \neq 0$ respectively:

$$
\begin{aligned}
& w_{1_{1}}(\eta)=\alpha_{0}-\frac{2 h}{A}\left(d^{2} \omega+B d-E\right) \times\left(d+\frac{B}{2 \omega}+\frac{\sqrt{\Omega}}{2 \omega} \operatorname{coth}\left(\frac{\sqrt{\Omega}}{2 A} \eta\right)\right)^{-1} . \\
& w_{1_{2}}(\eta)=\alpha_{0}-\frac{2 h}{A}\left(d^{2} \omega+B d-E\right) \times\left(d+\frac{B}{2 \omega}+\frac{\sqrt{\Omega}}{2 \omega} \tanh \left(\frac{\sqrt{\Omega}}{2 A} \eta\right)\right)^{-1} .
\end{aligned}
$$

Substituting Eq. (16) into Eq. (15), along with Eq. (8) and simplifying, our exact solutions become, if $C_{1}=0$ but $C_{2} \neq 0$; $C_{2}=0$ but $C_{1} \neq 0$ respectively:

$$
\begin{gathered}
w_{1_{3}}(\eta)=\alpha_{0}-\frac{2 h}{A}\left(d^{2} \omega+B d-E\right) \times\left(d+\frac{B}{2 \omega}+\frac{\sqrt{-\Omega}}{2 \omega} \cot \left(\frac{\sqrt{-\Omega}}{2 A} \eta\right)\right)^{-1} . \\
w_{1_{4}}(\eta)=\alpha_{0}-\frac{2 h}{A}\left(d^{2} \omega+B d-E\right) \times\left(d+\frac{B}{2 \omega}+\frac{\sqrt{-\Omega}}{2 \omega} \tan \left(\frac{\sqrt{-\Omega}}{2 A} \eta\right)\right)^{-1} .
\end{gathered}
$$

Substituting Eq. (16) into Eq. (15), together with Eq. (9) and simplifying, our obtained solution becomes:

$$
w_{1_{5}}(\eta)=\alpha_{0}-\frac{2 h}{A}\left(d^{2} \omega+B d-E\right) \times\left(d+\frac{B}{2 \omega}+\frac{C_{2}}{C_{1}+C_{2} \eta}\right)^{-1} .
$$

Substituting Eq. (16) into Eq. (15), along with Eq. (10) and simplifying, we obtain following traveling wave solutions, if $C_{1}=0$ but $C_{2} \neq 0 ; C_{2}=0$ but $C_{1} \neq 0$ respectively: 


$$
\begin{gathered}
w_{1_{6}}(\eta)=\alpha_{0}-\frac{2 h}{A}\left(d^{2} \omega+B d-E\right) \times\left(d+\frac{\sqrt{\Delta}}{\omega} \operatorname{coth}\left(\frac{\sqrt{\Delta}}{A} \eta\right)\right)^{-1} . \\
w_{1_{7}}(\eta)=\alpha_{0}-\frac{2 h}{A}\left(d^{2} \omega+B d-E\right) \times\left(d+\frac{\sqrt{\Delta}}{\omega} \tanh \left(\frac{\sqrt{\Delta}}{A} \eta\right)\right)^{-1} .
\end{gathered}
$$

Substituting Eq. (16) into Eq. (15), together with Eq. (11) and simplifying, our obtained exact solutions become, if $C_{1}=0$ but $C_{2} \neq 0 ; C_{2}=0$ but $C_{1} \neq 0$ respectively:

$$
\begin{aligned}
& w_{1_{8}}(\eta)=\alpha_{0}-\frac{2 h}{A}\left(d^{2} \omega+B d-E\right) \times\left(d+\frac{\sqrt{-\Delta}}{\omega} \cot \left(\frac{\sqrt{-\Delta}}{A} \eta\right)\right)^{-1} \\
& w_{1_{9}}(\eta)=\alpha_{0}-\frac{2 h}{A}\left(d^{2} \omega+B d-E\right) \times\left(d-\frac{\sqrt{-\Delta}}{\omega} \tan \left(\frac{\sqrt{-\Delta}}{A} \eta\right)\right)^{-1},
\end{aligned}
$$

where $\eta=x+\frac{1}{A}\left(h B+2 h d \omega-a_{0} A\right) t$.

Again for set 2, substituting Eq. (17) into Eq. (15), along with Eq. (7) and simplifying, our traveling wave solutions become, if $C_{1}=0$ but $C_{2} \neq 0 ; C_{2}=0$ but $C_{1} \neq 0$ respectively:

$$
\begin{gathered}
w_{2_{1}}(\eta)=\alpha_{0}+\frac{1}{A}\left(h(B+2 d \omega)+h \sqrt{\Omega} \operatorname{coth}\left(\frac{\sqrt{\Omega}}{2 A} \eta\right)\right), \\
w_{2_{2}}(\eta)=\alpha_{0}+\frac{1}{A}\left(h(B+2 d \omega)+h \sqrt{\Omega} \tanh \left(\frac{\sqrt{\Omega}}{2 A} \eta\right)\right),
\end{gathered}
$$

Substituting Eq. (17) into Eq. (15), along with Eq. (8) and simplifying yields exact solutions, if $C_{1}=0$ but $C_{2} \neq 0$; $C_{2}=0$ but $C_{1} \neq 0$ respectively:

$$
\begin{gathered}
w_{2_{3}}(\eta)=\alpha_{0}+\frac{1}{A}\left(h(B+2 d \omega)+i h \sqrt{\Omega} \cot \left(\frac{\sqrt{-\Omega}}{2 A} \eta\right)\right), \\
w_{2_{4}}(\eta)=\alpha_{0}+\frac{1}{A}\left(h(B+2 d \omega)-i h \sqrt{\Omega} \tan \left(\frac{\sqrt{-\Omega}}{2 A} \eta\right)\right) .
\end{gathered}
$$

Substituting Eq. (17) into Eq. (15), along with Eq. (9) and simplifying, our obtained solution becomes:

$$
w_{25}(\eta)=\alpha_{0}+\frac{1}{A}\left(h(B+2 d \omega)+2 h \omega\left(\frac{C_{2}}{C_{1}+C_{2} \eta}\right)\right)
$$

Substituting Eq. (17) into Eq. (15), together with Eq. (10) and simplifying, yields following traveling wave solutions, if $C_{1}=0$ but $C_{2} \neq 0 ; C_{2}=0$ but $C_{1} \neq 0$ respectively:

$$
\begin{gathered}
w_{2_{6}}(\eta)=\alpha_{0}+\frac{1}{A}\left(2 h \omega d+2 h \sqrt{\Delta} \operatorname{coth}\left(\frac{\sqrt{\Delta}}{A} \eta\right)\right) . \\
w_{2_{7}}(\eta)=\alpha_{0}+\frac{1}{A}\left(2 h \omega d+2 h \sqrt{\Delta} \tanh \left(\frac{\sqrt{\Delta}}{A} \eta\right)\right) .
\end{gathered}
$$

Substituting Eq. (17) into Eq. (15), along with Eq. (11) and simplifying, our exact solutions become, if $C_{1}=0$ but $C_{2} \neq 0$; $C_{2}=0$ but $C_{1} \neq 0$ respectively:

$$
\begin{gathered}
w_{2_{8}}(\eta)=\alpha_{0}+\frac{1}{A}\left(2 h \omega d+2 i h \sqrt{\Delta} \cot \left(\frac{\sqrt{-\Delta}}{A} \eta\right)\right) \\
w_{2_{9}}(\eta)=\alpha_{0}+\frac{1}{A}\left(2 h \omega d-2 i h \sqrt{\Delta} \tan \left(\frac{\sqrt{-\Delta}}{A} \eta\right)\right)
\end{gathered}
$$

where $\eta=x-\frac{1}{A}\left(a_{0} A+h B+2 h d \omega\right) t$.

Similarly, for set 3, substituting Eq. (18) into Eq. (15), together with Eq. (7) and simplifying, yields following traveling wave 
solutions, if $C_{1}=0$ but $C_{2} \neq 0 ; C_{2}=0$ but $C_{1} \neq 0$ respectively:

$$
\begin{aligned}
& w_{3_{1}}(\eta)=\alpha_{0}+\frac{1}{A}\left(h \sqrt{\Omega} \times \operatorname{coth}\left(\frac{\sqrt{\Omega}}{2 A} \eta\right)+\frac{2 h}{\sqrt{\Omega}}\left(4 E \omega+B^{2}\right) \times \tanh \left(\frac{\sqrt{\Omega}}{2 A} \eta\right)\right) . \\
& w_{3_{2}}(\eta)=\alpha_{0}+\frac{1}{A}\left(h \sqrt{\Omega} \times \tanh \left(\frac{\sqrt{\Omega}}{2 A} \eta\right)+\frac{2 h}{\sqrt{\Omega}}\left(4 E \omega+B^{2}\right) \times \operatorname{coth}\left(\frac{\sqrt{\Omega}}{2 A} \eta\right)\right) .
\end{aligned}
$$

Substituting Eq. (18) into Eq. (15), along with Eq. (8) and simplifying, we obtain following solutions, if $C_{1}=0$ but $C_{2} \neq 0$; $C_{2}=0$ but $C_{1} \neq 0$ respectively:

$$
\begin{aligned}
& w_{3_{3}}(\eta)=\alpha_{0}+\frac{1}{A}\left(h i \sqrt{\Omega} \times \cot \left(\frac{\sqrt{-\Omega}}{2 A} \eta\right)+\frac{2 h}{i \sqrt{\Omega}}\left(4 E \omega+B^{2}\right) \times \tan \left(\frac{\sqrt{-\Omega}}{2 A} \eta\right)\right) . \\
& w_{3_{4}}(\eta)=\alpha_{0}-\frac{1}{A}\left(h i \sqrt{\Omega} \times \tan \left(\frac{\sqrt{-\Omega}}{2 A} \eta\right)+\frac{2 h}{i \sqrt{\Omega}}\left(4 E \omega+B^{2}\right) \times \cot \left(\frac{\sqrt{-\Omega}}{2 A} \eta\right)\right) .
\end{aligned}
$$

Substituting Eq. (18) into Eq. (15), along with Eq. (9) and simplifying, our obtained solution becomes:

$$
w_{3_{5}}(\eta)=\alpha_{0}+\frac{2 h \omega}{A} \times\left(\frac{C_{2}}{C_{1}+C_{2} \eta}\right)+\frac{h}{A \omega}\left(4 E \omega+B^{2}\right) \times\left(\frac{C_{2}}{C_{1}+C_{2} \eta}\right)^{-1}
$$

Substituting Eq. (18) into Eq. (15), along with Eq. (10) and simplifying, yields following exact traveling wave solutions, if $C_{1}=0$ but $C_{2} \neq 0 ; C_{2}=0$ but $C_{1} \neq 0$ respectively:

$$
\begin{aligned}
& w_{3_{6}}(\eta)=\alpha_{0}+\frac{2 h \omega}{A} \times\left(\frac{-B}{2 \omega}+\frac{\sqrt{\Delta}}{\omega} \operatorname{coth}\left(\frac{\sqrt{\Delta}}{A} \eta\right)\right)+\frac{h}{A \omega}\left(4 E \omega+B^{2}\right) \times\left(\frac{-B}{2 \omega}+\frac{\sqrt{\Delta}}{\omega} \operatorname{coth}\left(\frac{\sqrt{\Delta}}{A} \eta\right)\right)^{-1} . \\
& w_{3_{7}}(\eta)=a_{0}+\frac{2 h \omega}{A} \times\left(\frac{-B}{2 \omega}+\frac{\sqrt{\Delta}}{\omega} \tanh \left(\frac{\sqrt{\Delta}}{A} \eta\right)\right)+\frac{h}{A \omega}\left(4 E \omega+B^{2}\right) \times\left(\frac{-B}{2 \omega}+\frac{\sqrt{\Delta}}{\omega} \tanh \left(\frac{\sqrt{\Delta}}{A} \eta\right)\right)^{-1} .
\end{aligned}
$$

Substituting Eq. (18) into Eq. (15), along with Eq. (11) and simplifying, our obtained exact solutions become, if $C_{1}=0$ but $C_{2} \neq 0 ; C_{2}=0$ but $C_{1} \neq 0$ respectively:

$$
\begin{aligned}
& w_{3_{8}}(\eta)=\alpha_{0}+\frac{2 h \omega}{A} \times\left(\frac{-B}{2 \omega}+\frac{\sqrt{-\Delta}}{\omega} \cot \left(\frac{\sqrt{-\Delta}}{A} \eta\right)\right)+\frac{h}{A \omega}\left(4 E \omega+B^{2}\right) \times\left(\frac{-B}{2 \omega}+\frac{\sqrt{-\Delta}}{\omega} \cot \left(\frac{\sqrt{-\Delta}}{A} \eta\right)\right)^{-1} \\
& w_{3_{9}}(\eta)=\alpha_{0}+\frac{2 h \omega}{A} \times\left(\frac{-B}{2 \omega}-\frac{\sqrt{-\Delta}}{\omega} \tan \left(\frac{\sqrt{-\Delta}}{A} \eta\right)\right)+\frac{h}{A \omega}\left(4 E \omega+B^{2}\right) \times\left(\frac{-B}{2 \omega}-\frac{\sqrt{-\Delta}}{\omega} \tan \left(\frac{\sqrt{-\Delta}}{A} \eta\right)\right)^{-1}
\end{aligned}
$$

where $\eta=x-a_{0} t$.

\subsection{Results and Discussion}

It is worth declaring that some of our obtained solutions are in good agreement with already published results which are presented in the following table. 
Table 1. Comparison between Neyrame et al. [27] solutions and our solutions

\begin{tabular}{|c|c|}
\hline Neyrame et al. [27] solutions & Obtained solutions \\
\hline $\begin{array}{l}\text { i. If } C_{1}=0 \text { and } u(\xi)=w_{2_{1}}(\eta) \text {, solutions Eq. (16) becomes: } \\
\qquad w_{2_{1}}(\eta)=2 h\left(\lambda^{2}-4 \mu\right) \times \operatorname{coth}^{2}\left(\frac{\sqrt{\lambda^{2}-4 \mu \eta}}{2}\right)-\frac{\lambda}{2}+\alpha_{0}\end{array}$ & $\begin{array}{c}\text { i. If } A=1, C=0, \Omega=\lambda^{2}-4 \mu, B=1, h=2 h, \\
d=-\frac{\lambda}{4 h}, B=0 \text { then the solution is } \\
w_{2_{1}}(\eta)=2 h\left(\lambda^{2}-4 \mu\right) \times \operatorname{coth}^{2}\left(\frac{\sqrt{\lambda^{2}-4 \mu \eta}}{2}\right)-\frac{\lambda}{2}+\alpha_{0} .\end{array}$ \\
\hline $\begin{array}{l}\text { ii. If } C_{1}=0 \text { and } u(\xi)=w_{2_{3}}(\eta) \text {, solutions Eq. (16) becomes: } \\
\qquad w_{2_{3}}(\eta)=2 h\left(4 \mu-\lambda^{2}\right) \times \cot ^{2}\left(\frac{\sqrt{4 \mu-\lambda^{2}} \eta}{2}\right)-\frac{\lambda}{2}+\alpha_{0}\end{array}$ & $\begin{array}{l}\text { ii. If } A=1, \quad C=0, \Omega=\lambda^{2}-4 \mu, B=1, h=2 h, \\
d=-\frac{\lambda}{4 h}, B=0 \text { then the solution is } \\
w_{2_{3}}(\eta)=2 h\left(4 \mu-\lambda^{2}\right) \times \cot ^{2}\left(\frac{\sqrt{4 \mu-\lambda^{2}} \eta}{2}\right)-\frac{\lambda}{2}+\alpha_{0} .\end{array}$ \\
\hline $\begin{array}{l}\text { iii. If } u(\xi)=w_{2_{5}}(\eta) \text {, solutions Eq. (16) becomes: } \\
\qquad w_{2_{5}}(\eta)=2 h\left(\frac{C_{2}}{C_{1}+C_{2} \eta}\right)\end{array}$ & $\begin{array}{l}\text { iii. If } A=1, C=0, B=-2 d \text { and } \alpha_{0}=0 \text { then the } \\
\text { solution is } \\
w_{2_{5}}(\eta)=2 h\left(\frac{C_{2}}{C_{1}+C_{2} \eta}\right) .\end{array}$ \\
\hline
\end{tabular}

Beside this table, we obtain more new exact traveling wave solutions $w_{2_{2}}(\eta), w_{2_{4}}(\eta), w_{2_{6}}(\eta)-w_{2_{9}}(\eta), w_{1_{1}}(\eta)-w_{1_{9}}(\eta), w_{3_{1}}(\eta)-w_{3_{9}}(\eta)$ in this article, which have not been reported in the previous literature.

\subsection{Graphical Representation of the Solutions}

The graphical illustrations of the solutions are given below in the figures with the aid of Maple.

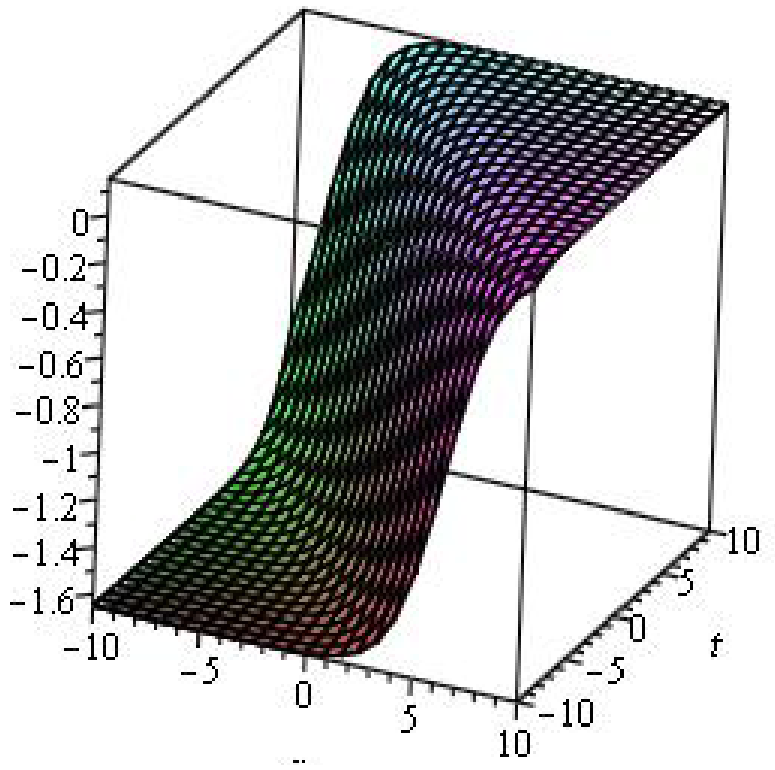

Figure 1. Modulus plot of Kink wave, Shape of $w_{1_{2}}(\eta)$ when $A=4$, $B=1, \quad C=1, E=1, \alpha_{0}=1, d=1, h=1$ and $-10 \leq x, t \leq 10$.

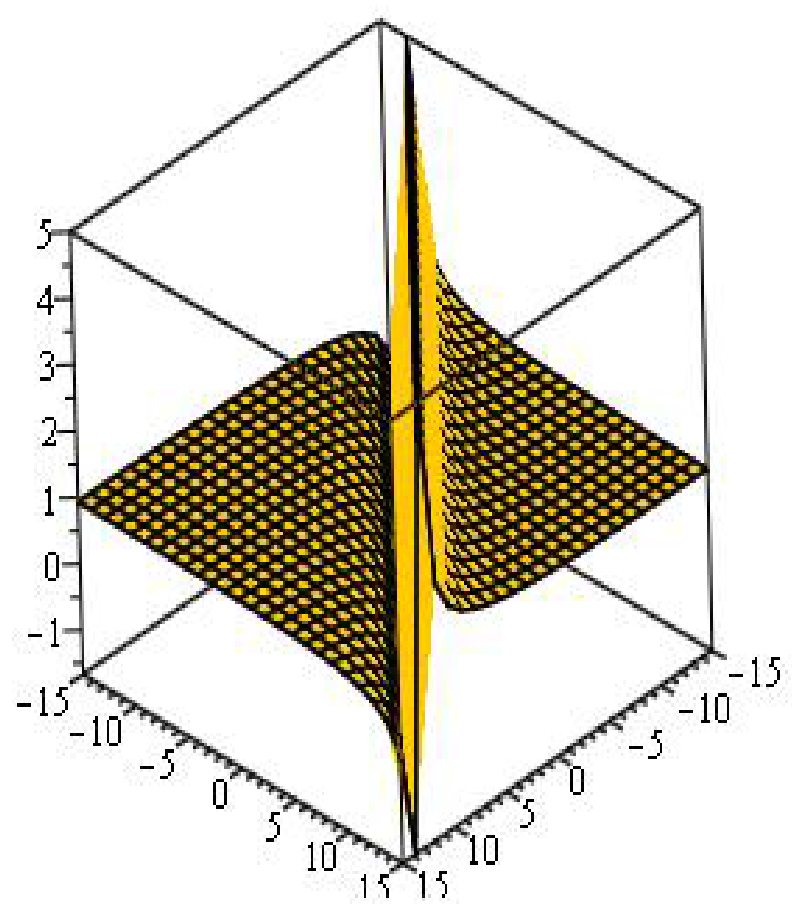

Figure 2. Modulus plot of singular Kink wave, Shape of $w_{2_{5}}(\eta)$ when $A=1, \quad B=2, C=2, E=1, d=1, h=1, \quad \alpha_{0}=1, \quad C_{2}=1$, $C_{1}=2$, and $-15 \leq x, t \leq 15$. 


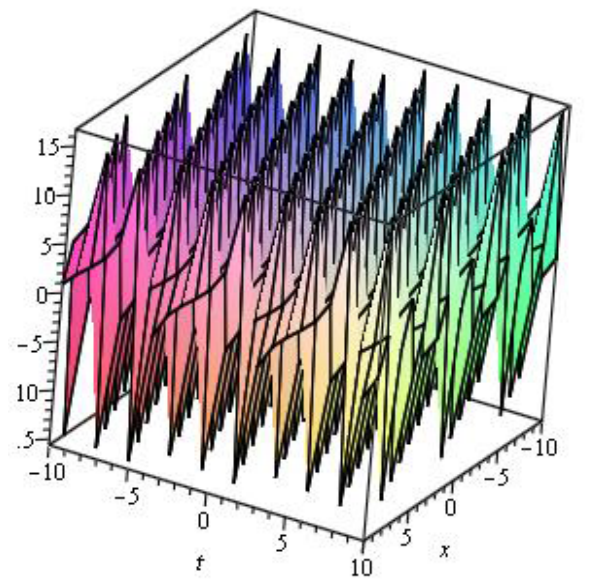

Figure 3. Modulus plot periodic wave, Shape of $w_{1_{8}}(\eta)$ when $A=1$, $B=0, \quad C=2, \quad E=2, \quad d=1, \quad h=1, \quad \alpha_{0}=1, \quad$ and $-10 \leq x, t \leq 10$.

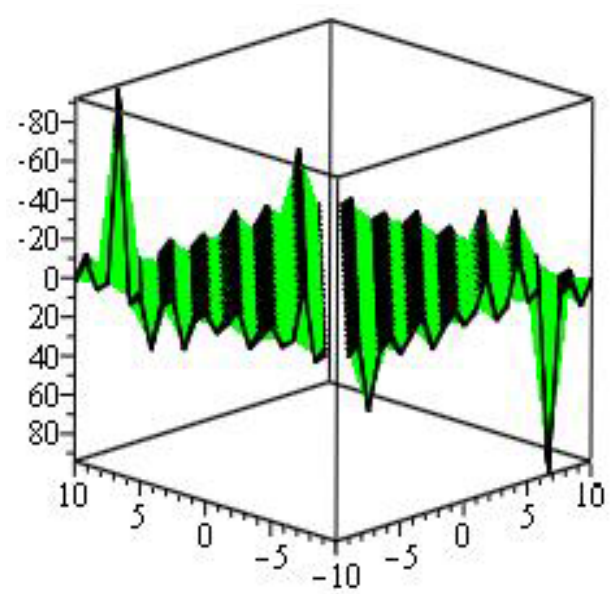

Figure 4. Modulus plot singular periodic wave, Shape of $w_{3_{3}}(\eta)$ when $A=2, B=1, C=4, E=1, V=1, h=1, \alpha_{0}=1$ and $-10 \leq x, t \leq 10$.

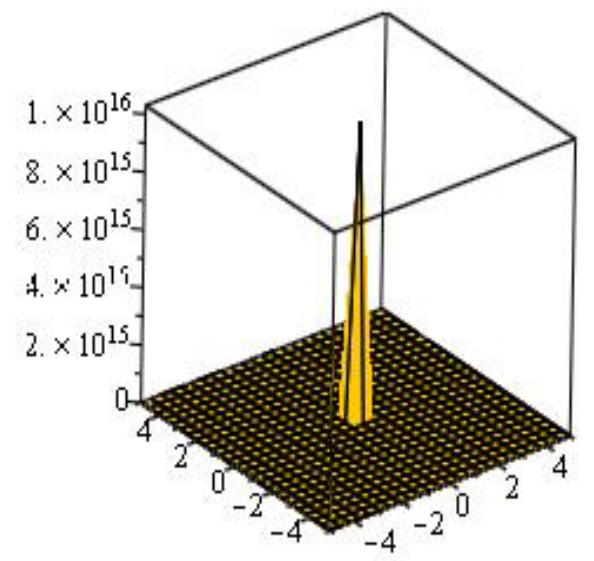

Figure 5. Modulus plot of single soliton wave, Shape of $w_{2_{1}}(\eta)$ when $A=4, B=1, C=1, E=1, d=1, h=1, \quad \alpha_{0}=1$ and $-5 \leq x, t \leq 5$.

The solutions corresponding to $w_{1_{1}}(\eta), \quad w_{1_{5}}(\eta), \quad w_{1_{6}}(\eta)$, $w_{2_{5}}(\eta), \quad w_{2_{8}}(\eta), \quad w_{3_{1}}(\eta), \quad w_{3_{2}}(\eta), \quad w_{3_{5}}(\eta)-w_{3_{7}}(\eta) \quad$ is identical to the solution $w_{2_{5}}(\eta)$, the solution corresponding to $w_{1_{7}}(\eta), w_{2_{2}}(\eta), w_{2_{7}}(\eta)$ is identical to the solution $w_{1_{2}}(\eta)$, the solution corresponding to $w_{1_{3}}(\eta), w_{1_{4}}(\eta)$ $w_{1_{8}}(\eta), \quad w_{1_{9}}(\eta), \quad w_{2_{3}}(\eta), \quad w_{2_{4}}(\eta), \quad w_{2_{6}}(\eta), \quad w_{2_{9}}(\eta) \quad$ is identical to the solution $w_{1_{8}}(\eta)$ and the solution corresponding to $w_{3_{4}}(\eta), w_{3_{8}}(\eta), w_{3_{9}}(\eta)$ is identical to the solution $w_{3_{3}}(\eta)$.

\section{Conclusion}

In this study, we considered the Benjamin-Ono equation. We apply the new approach of generalized $\left(G^{\prime} / G\right)$ -expansion method for the exact solution of this equation and constructed some new solutions which are not found in the previous literature. The method offers solutions with free parameters that might be imperative to explain some intricate physical phenomena. This study shows that the new generalized $\left(G^{\prime} / G\right)$-expansion method is quite efficient and practically well suited to be used in finding exact solutions of NLEEs. Also, we observe that the new generalized $\left(G^{\prime} / G\right)$ -expansion method is straightforward and can be applied to many other nonlinear evolution equations.

\section{REFERENCES}

[1] E. G. Fan, Two new applications of the homogeneous balance method. Phys. Lett. A. 265 (2000) 353-357.

[2] A. M. Wazwaz, The tanh method for generalized forms of nonlinear heat conduction and Burgers-Fisher equations, Appl. Math.Comput. 169 (2005) 321-338.

[3] E. Fan, Extended tanh-function method and its applications to nonlinear equations. Phys Lett A 277(4- 5) (2000) 212-218

[4] S. A. El-Wakil, M. A. Abdou, New exact travelling wave solutions using modified extended tanh-function method. Chaos Solitons Fractals 31(4) (2007) 840-852.

[5] J. H. He and X. H. Wu, Exp-function method for nonlinear wave equations, Chaos, Solitons Fract. 30 (2006) 700-708.

[6] M. A. Akbar and N. H. M. Ali, New Solitary and Periodic Solutions of Nonlinear Evolution Equation by Exp-function Method, World Appl. Sci. J., 17(12) (2012) 1603-1610.

[7] H. Naher, A. F. Abdullah and M. A. Akbar, New traveling wave solutions of the higher dimensional nonlinear partial differential equation by the Exp-function method, J. Appl. Math., Article ID 575387 (2012) 14 pages. doi: $10.1155 / 2012 / 575387$.

[8] A. M. Wazwaz, A sine-cosine method for handle nonlinear wave equations, Applied Mathematics and Computer Modeling, 40 (2004) 499-508. 
[9] M. Usman, A. Nazir, T. Zubair, I. Rashid, Z. Naheed, S.T. Mohyud-Din, Solitary wave solutions of (3+1)-dimensional Jimbo Miwa and Pochhammer-Chree equations by modified Exp-function method, Int. J. Modern Math. Sci. 5(1) (2013) 27-36.

[10] Z. Yan and H. Zhang, New explicit solitary wave solutions and periodic wave solutions for Whitham Broer-Kaup equation in shallow water, Physics Letters. A, vol. 285 no.5-6 (2001) 355-362.

[11] D. Liu, Jacobi elliptic function solutions for two variant Boussinesq equations, Chaos solitons Fractals, 24 (2005) 1373-85.

[12] Y. Chen and Q. Wang, Extended Jacobi elliptic function rational expansion method and abundant families of Jacobi elliptic functions solutions to $(1+1)$-dimensional dispersive long wave equation, Chaos solitons Fractals, 24 (2005) 745-57.

[13] A. M. Wazwaz, Multiple-soliton solutions for a $(3+1)$-dimensional generalized KP equation, Commun Nonlinear Sci Numer Simulat 17 (2012) 491-495.

[14] T. L. Bock and M. D. Kruskal, A two-parameter Miura transformation of the Benjamin-One equation, Phys. Lett. A 74 (1979) 173-176.

[15] M. L. Wang, X. Z. Li and J. Zhang, The $\left(G^{\prime} / G\right)$-expansion method and traveling wave solutions of nonlinear evolution equations in mathematical physics, Phys. Lett. A, 372 (2008) 417-423.

[16] A. Bekir, Application of the $\left(G^{\prime} / G\right)$-expansion method for nonlinear evolution equations, Phys. Lett. A 372 (2008) 3400-3406.

[17] E. M. E. Zayed, The $\left(G^{\prime} / G\right)$-expansion method and its applications to some nonlinear evolution equations in the mathematical physics, J. Appl. Math. Comput., 30 (2009) 89-103.

[18] S. Zhang, J. Tong and W. Wang, A generalized $\left(G^{\prime} / G\right)$ -expansion method for the $\mathrm{mKdV}$ equation with variable coefficients, Phys. Lett. A, 372 (2008) 2254-2257.

[19] M. A. Akbar, N. H. M. Ali and E. M. E. Zayed, A generalized and improved $\left(G^{\prime} / G\right)$-expansion method for nonlinear evolution equations, Math. Prob. Engr., Vol. 2012 (2012) 22 pages. doi: 10.1155/2012/459879.

[20] M. N. Alam, M. A. Akbar and S. T. Mohyud-Din, A novel $\left(G^{\prime} / G\right)$-expansion method and its application to the Boussinesq equation, Chin. Phys. B, Article ID 131006 (accepted for publication).

[21] M. N. Alam and M. A. Akbar, Traveling wave solutions of the nonlinear (1+1)-dimensional modified Benjamin-Bona-Mahony equation by using new $\left(G^{\prime} / G\right)$ -expansion, Physical Review \& Research International (Sciencedomain international), Manuscript number: 2013_PRRI_5710,2013(accepted for publication).

[22] A. J. M. Jawad, M. D. Petkovic and A. Biswas, Modified simple equation method for nonlinear evolution equations, Appl. Math. Comput., 217 (2010) 869-877.

[23] K. Khan, M. A. Akbar and M. N. Alam, Traveling wave solutions of the nonlinear Drinfel'd-Sokolov-Wilson equation and modified Benjamin-Bona-Mahony equations, J. Egyptian Math. Soc., DOI org/10.1016/j.joems.2013.04.010 (in press).

[24] J. Zhang, F. Jiang and X. Zhao, An improved $\left(G^{\prime} / G\right)$ -expansion method for solving nonlinear evolution equations, Int. J. Com. Math., 87(8) (2010) 1716-1725.

[25] M. J. Ablowitz and P. A. Clarkson, Soliton, nonlinear evolution equations and inverse scattering (Cambridge University Press, New York, 1991).

[26] H. Naher and F. A. Abdullah, New approach of $\left(G^{\prime} / G\right)$ -expansion method and new approach of generalized $\left(G^{\prime} / G\right)$-expansion method for nonlinear evolution equation, AIP Advances 3, 032116 (2013) DOI: 10. 1063/1.4794947.

[27] A. Neyrame, A. Roozi, S. S .Hosseini, S. M. Shafiof, Exact travelling wave solutions for some nonlinear partial differential equations, journal of King Saud University (Science), (2010), 22, 275-278. 